\title{
Ectodermal dysplasia-intellectual disability-central nervous system malformation syndrome
}

INSERM

\section{Source}

INSERM. (1999). Orphanet: an online rare disease and orphan drug data base. Ectodermal dysplasia-intellectual disability-central nervous system malformation syndrome. ORPHA:1812

Ectodermal dysplasia-intellectual disability-central nervous system malformation syndrome is a rare, multiple developmental anomalies syndrome characterized by the triad of ectodermal dysplasia (mostly hypohidrotic with dry skin and reduced sweating and sparse, fair scalp hair, eyebrows and eyelashes), severe intellectual disability and variable central nervous system anomalies (cerebellar hypoplasia, dilatation of ventricles, corpus callosum agenesis, Dandy-Walker malformation). Distinct craniofacial dysmorphism with macrocephaly, frontal bossing, midfacial hypoplasia and high arched or cleft palate, as well as cryptorchidism, feeding difficulties and hypotonia, are associated. There have been no further descriptions in the literature since 1998. 